\title{
COMMENT
}

\section{Answer to Rutger de Wit's comments to the proposed tentative "Ecological Law of Thermodynamics" (ELT)}

\author{
SVEN ERIK JØRGENSEN \\ Institute A, Environmental Chemistry, University Park 2, 2100 Copenhagen East, Denmark. E-mail: sej@dfuni.dk
}

Let me first of all thank Rutger de Wit for his comments and criticism of the proposed ELT, because it is important that we discuss ecological theory much more widely than it has been the case up to now. I assert that we have an ecosystem theory, when we put together the contributions from several system ecologists (included the contributions from late professor Ilya Prigogine and Ramon Margalef). The theory may not be complete and will most probably be improved significantly during the coming decades, but it is in my opinion sufficiently complete to be applied to explain ecological observations and in environmental management discussions. By application of the theory it becomes more obvious where it has short-comings. It is therefore crucial to discuss such an important hypothesis as ELT and propose improvements in the formulation and interpretation. So, let me try to answer the criticism and that may hopefully together with Rutger de Wit's comments contribute to an even better formulation and interpretation of ELT.

I have used exergy to express the working capacity content of ecosystems. Exergy is, as correctly indicated by Rutger de Wit, the energy that can do work relatively to the environment. What is the environment of an ecosystem? Of course other ecosystems; but if we would use adjacent ecosystems as reference state, we would just get the difference in exergy between two ecosystems and it would not be interesting. So, I used the same ecosystem at the same temperature and pressure but at thermodynamic equilibrium as reference state to get an expression for the distance from thermodynamic equilibrium, because that would be a good measure of the development of an ecosystem. I have lately applied the term eco-exergy to emphasize that it is not the technological exergy that uses the environment as reference state. We can calculate the eco-exergy of an ecosystem compared with the same system at the same temperature and pressure but in form of an inorganic soup without any life, biological structure, information or organic molecules as follows:

$$
E x=R T \sum_{i=0}^{i=n} C_{i} \ln C_{i} / C_{i, o}
$$

where $\mathrm{R}$ is the gas constant $(8.317 \mathrm{~J} / \mathrm{K}$ moles $=$ 0.08207 liter. atm / $\mathrm{K}$ moles), $\mathrm{T}$ is the temperature of the environment, while $C_{i}$ is the concentration of the i'th component expressed in a suitable unit, e.g. for phytoplankton in a lake $C_{i}$ could be expressed as mg $/ 1$ or as $\mathrm{mg} / \mathrm{l}$ of a focal nutrient. $C_{i, o}$ is the concentration of the $i$ 'th component at thermodynamic equilibrium and $\mathrm{n}$ is the number of components. Notice that $\ln$ (concentration) is applied as the chemical potential. $C_{i, o}$ is of course a very small concentration (except for $i=0$, which is considered to cover the inorganic compounds), corresponding to a very low probability of forming complex organic compounds spontaneously in an inorganic soup at 
thermodynamic equilibrium. $C_{i, o}$ is even lower for the various organisms, because the probability of forming the organisms is very low with their embodied information which implies that the genetic code should be correct.

It is possible to distinguish between the exergy of information and of biomass. $p_{i}$ defined as $c_{i} / A$, where

$$
A=\sum_{i=1}^{n} c_{i}
$$

is the total amount of matter in the system, is introduced as new variable in equation:

$$
E x=A R T \sum_{i=1}^{n} p_{i} \ln p_{i} / p_{i, o}+A \ln A / A_{o}
$$

As $A \approx A o$, exergy becomes a product of the total biomass $A$ (multiplied by $R T$ ) and

Kullback measure:

$$
K=\sum_{i=1}^{n} p_{i} \ln \left(p_{i} / p_{i, o}\right)
$$

where $p_{i}$ and $p_{i o}$ are probability distributions, a posteriori and a priori to an observation of the molecular detail of the system. It means that $K$ expresses the amount of information that is gained as a result of the observations. If we observe a system, which consists of two connected chambers, we expect the molecules to be equally distributed in the two chambers, i.e., $\mathrm{p}_{1}=\mathrm{p}_{2}$ is equal to $1 / 2$. If we, on the other hand, observe that all the molecules are in one chamber, we get $\mathrm{p}_{1}=1$ and $\mathrm{p}_{2}=0$. So, information can at least under some circumstances be used to perform work.

Rutger de Wit is of course right when he tells us that what I call eco-exergy is a difference in free energy. I prefer to call it eco-exergy, because every time I calculate eco-exergy for an ecosystem, I am using a new reference state and we are using all the time a difference in free energy. I can therefore agree with Rutger de Wit in his formulation of ELT, because he uses the expressions "the greatest dis- tance away from thermodynamic equilibirum" and "a flow of free energy through the system", which are completely consistent with my idea. If Rutger de Wit prefers that formulation and not to use eco-exergy because it is not necessary unless you discuss a specific case, then I can fully accept Rutger de Wit's formulation. It is however more easy in a specific case to ask: how much is the eco-exergy of ecosystem X? In stead of "What is the free energy of system $X$ relatively to the thermodynamic equilibrium of the same system at the same temperature and pressure? So much for the formulation.

I agree with Rutger de Wit that I don't include in my calculations the information of the ecological network; but I would like to include it. The information of an ecological network in our simplified description of the real very complex ecological network, following the ascendency calculations by Ulanowicz, is however very small and negligible compared with the information of the genes. I agree with Ramon Margalef's highlighting the historical process, but I am of the opinion that the composition and coding power of the genes reflect the historical process. The genes have all the time been developed because they were building on what was already achieved. Today we have a complete knowledge of the genetic information of eleven species, and it has been used to give new weighting factors. They have been published in "Calculations of exergy for organisms" Ecological Modelling 185: 153-164 (2005). Rutger de Wit is right: the previous values were underestimations of the information content, because the genes of particularly higher organisms code for more amino acids than the average that was previously applied in my calculations. The models where I have used the eco-exergy calculations have fortunately applied relative values that are approximately unchanged. The model results are therefore still (relatively) valid. Rutger de Wit proposes to call the amplification factors for "estimators of the distance from thermodynamic equilibrium" and I can agree that it may be a more correct name. 